\title{
Dynamic Voltage Stability Comparison of Thermal and Wind Power Generation with Different Static and Dynamic Load Models
}

\author{
Lina F. Acevedo, Gilbert Bothia-Vargas, John E. Candelo \\ Department of Electrical Energy and Automation, Universidad Nacional de Colombia, Sede Medellín, Colombia
}

\begin{tabular}{l}
\hline \hline Article Info \\
\hline Article history: \\
Received Jan 15, 2018 \\
Revised Mar 20, 2018 \\
Accepted Apr 11, 2018 \\
\hline
\end{tabular}

\section{Keyword:}

Continuation power flow Exponential recovery load Frequency-dependent load Induction motor ZIP load model

\begin{abstract}
This paper presents a static and dynamic voltage stability analysis of a power network with thermal and wind generation considering static and dynamic load models. The thermal plant was modeled as a synchronous machine and the wind farm as a variable speed induction generator based on a doubly-fed induction generator. The load considered the ZIP, exponential recovery, induction motor, and frequency-dependent load models. The bifurcation points were found by continuation power flow and sensitivity analyses. In addition, dynamic voltage stability assessments were performed considering changes in the moment of inertia and the frequency parameters. All simulations were carried out in a 4-bus power system and using the power system analysis toolbox (PSAT) and MATLAB script code. The results show that the thermal generator had difficulties to maintain stability under dynamic load variations and frequency changes, the wind generator had difficulties to maintain voltage for the load with induction motors, and both generators had difficulties when the moment of inertia is increased.
\end{abstract}

Copyright (c) 2018 Institute of Advanced Engineering and Science. All rights reserved.

\section{Corresponding Author:}

John E. Candelo,

Department of Electrical Energy and Automation,

Universidad Nacional de Colombia, Sede Medellín,

Carrera 80 No 65 - 223, Facultad de Minas, Barrio Robledo, Colombia.

Email: jecandelob@unal.edu.co

\section{INTRODUCTION}

Power systems are becoming more complex due to the continuous increase in power demand and expansion of electrical networks, the inclusion of various types of loads, and the integration of large renewable energies and power electronics. Thus, power system operation is subjected to continuous disturbance in the generation, transmission, and the load, which causes the reduction of operation margins and leads the system close to its stability limits and collapse [1], [2]. Therefore, studying the voltage stability (VS) of the power system with different types of generators and loads is required to evaluate the security of the network.

Some researchers have previously studied this problem. In [3], PV curves are used to study the integration of wind generation with compensation devices such as SVC in order to determine the maximum loadability of the system. In [4], the authors performed a probabilistic voltage stability-constrained optimal power flow to improve the voltage stability margin and social welfare. In [5], the authors studied the impact of variable-speed wind power generation in long-term VS while operating synchronous generators with dynamic reactive power capabilities. In [6], a VS-constrained optimal power flow was presented to determine the required loading margin. In [7], static and dynamic reactive power compensation are used to study the integration with generators, considering a $10 \%$ step disturbance in the dynamic load model. In [8], the integration of variable-speed wind turbines on long-term VS is studied; in this case, the capability curves, dynamic model of excitation limiters, on load tap changers, and static and dynamic loads were considered. In 
[9], a doubly-fed induction generator (DFIG) under maximum power-point tracking mode is used to find the control effect of load shedding, by sensitivities of $L$ indices in load buses. In [10], a Monte Carlo probabilistic methodology was used to evaluate the oscillatory stability margin in a power system with largescale wind generation. In [11], a voltage stability analysis is used to evaluate the effects produced in a power network when reactive power is injected in some selected busses. In [12], the performance of a DFIG driven by a wind turbine is evaluated using a control vector technique, obtaining that the use of a AC/DC/AC converter helped improving stability and reliability of the network. In [13], the optimal location of wind turbines in a power grid was considered to evaluate the performance of the network, where the mathematical formulation considered objective functions as the maximization of system loadability and the minimization of real power loss of transmission lines; besides, some constraints such as security and small signal stability were included in the formulation.

Most studies in the literature show that voltage stability is analyzed by using static load models. However, power systems are nonlinear and complex because demand and generation are composed of distinct types of elements that generate dynamic behavior, and their combination causes many uncertainties in the variation to be studied. There are still no exact models that demonstrate the actual behavior of the loads and with constant parameters it is difficult to predict the proximity to the voltage collapse of a power system that is subjected to several changes.

The main purpose of this paper is to study the voltage stability of a power system that considers the inclusion of thermal and wind power generation in a network with different static and dynamic load models. The aim is to compare the voltage behavior of the system under the maximum load and close to the saddlenode bifurcation for the different load models. A sensitivity analysis is performed by increasing the real and reactive power of the load and, after determining critical operation close to the voltage collapse, time-domain simulations are used to compare the interaction of different types of load with the thermal and wind generation. For this purpose, the rest of the paper has been organized as follows: Section 2 describes the generator models, load models, and the stability analysis techniques used in the research; Section 3 presents the power system used for the test, the results, and the analysis; and Section 4 summarizes the conclusions drawn from the study.

\section{METHODOLOGY}

Figure 1 presents the flowchart of the methodology used for the voltage stability analysis with the two types of generators and four types of load models. The procedure consists of integrating to a power grid, two generators based on thermal and wind energies, and then the load models, ZIP, ER, IM, and FD, are considered. Next, PV curves are calculated by using the continuation power flow for each type of generation and each type of load. Finally, time-domain simulations are run to compare the behavior of the power grid with the different operation scenarios.

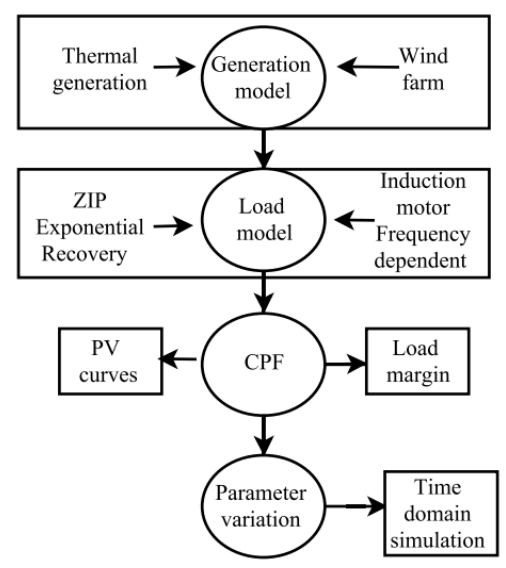

Figure 1. Flowchart of the methodology used for the research

\subsection{Generation model}

Most of the electrical generators in power systems are synchronous machines such as hydraulic and thermal generators. This is because the rotor uses DC excitation and imposes a synchronous characteristic 
that allows greater control of the voltage and frequency in the stator. In addition, these types of machines are preferred because they use the rotor as field windings to reduce the stress on the brushes as they operate with smaller current magnitudes than in generators where the armature is mobile. At the operational level, some of the most important variables in this machine are the active and reactive power, moment of inertia, and dynamic response parameters such as sub-transient, transient, and steady-state reactances. To evaluate the impact of load variations on the generator, these variables limit the voltage and current response at the system buses.

\subsubsection{Thermal generator}

Thermal generators are synchronous machines characterized by having a round rotor. The speed is greater than that of the salient-pole machine. Therefore, the mass and inertia of these machines are commonly greater than other machines, resulting in a longer mechanical start time and being more stable or slow forward response against changes produced in the network. Generators are commonly modeled with the d-q representation to facilitate the decoupling of the effects between stator and rotor. This allows to model the machine related to its windings with an open circuit test and determine the synchronous impedance. Other complementary tests such as stand still time response (SSTR) and slip test allow the characterization of the transient and mechanical characteristics of the d-q model [14].

The thermal generator was modeled as a four order synchronous machine. This type of machine can be formulated using the subtransient $\mathrm{d}$-axis voltage $e_{d}^{\prime \prime}$ instead of $e_{d}^{\prime}$. The differential equation is shown in (1):

$$
e_{d}^{\prime \prime}=\frac{\left(-e_{d}^{\prime \prime}+\left(x_{q}-x_{q}^{\prime \prime}\right) i_{q}\right)}{T_{q 0}^{\prime \prime}}
$$

where $T_{q 0}^{\prime \prime}$ is the subtransient time constant of the q-axis in open circuit, $x_{q}$ is the synchronous reactance of the q-axis, $x_{q}^{\prime \prime}$ is the subtransient reactance of the q-axis, $i_{q}$ represents the quadrature current, and $e_{d}^{\prime \prime}$ is the subtransient voltage of the d-axis.

\subsubsection{Wind generator}

Wind generators use different types of turbines, such as constant speed turbines, variable speed turbines (DFIG), and complements between the previous ones [15]. The DFIG facilitates the speed control, it is used to damp oscillations of the system caused by the load dynamics in the network or operational conditions whose bifurcations generate voltage collapses. It is necessary to verify the wind penetration through the dynamic analysis while considering different loading conditions and variations in its parameters [16]. The pitch angle control of DFIG is shown in (2):

$$
\dot{\theta_{p}}=\frac{\left(K_{p} \emptyset\left(w_{m}-w_{r e f}\right)-\theta_{p}\right)}{T_{p}}
$$

where $T_{p}$ is the pitch control time constant, $\emptyset$ is the parameter that changed the pitch angle set when $w_{m}-$ $w_{\text {ref }}$ exceeds the value $\pm \Delta w$ and $K_{p}$ is the pitch control gain.

\subsection{Load model}

There are two major groups for classifying loads: static and dynamic models. The static models focus on calculating the updated values of real and reactive powers, which have dependence on voltage and frequency values. This category includes the exponential, polynomial, and frequency-dependent models. These models must take into account the voltage levels and the changes in the topology of the network that affect the characteristics of load behavior. On the other hand, dynamic models are nonlinear such as exponential recovery and induction motors models and they can create many variations in the power system operation.

\subsubsection{ZIP load model}

The ZIP model can represent real and reactive powers of load as shown in (3) and (4). These equations are divided into the represented constant impedance (Z), constant current (I), and constant power load (P) [17]. The terms $V_{0}, P_{0}$, and $Q_{0}$ are the nominal values of voltage, real power, and reactive power, respectively, as determined under normal operating conditions of the power system. Moreover, coefficients $a$ and $b$ are used to represent each of type load, where $a_{l}$ and $b_{1}$ are constants used to represent the percentage of impedance in the model, $a_{2}$ and $b_{2}$ are constants used to represent the percentage of current in the model, and $a_{3}$ and $b_{3}$ are constants used to represent the percentage of real and reactive powers, respectively: 


$$
\begin{aligned}
& P_{\text {zip }}=P_{0}\left[a_{1}\left(\frac{V}{V_{0}}\right)^{2}+a_{2}\left(\frac{V}{V_{0}}\right)+a_{3}\right], \\
& Q_{\text {zip }}=Q_{0}\left[b_{1}\left(\frac{V}{V_{0}}\right)^{2}+b_{2}\left(\frac{V}{V_{0}}\right)+b_{3}\right] .
\end{aligned}
$$

\subsubsection{Exponential recovery $($ ER) model}

The ER model and its critical effect on voltage stability were studied in [18]. The model represents a nonlinear equation that depends on voltage as shown in Equations (5) to (8). Herein, the terms $V_{0}$ and $P_{0}$ represent, respectively, the initial voltage and power before a voltage change, $x_{p}$ is the active power recovery, $P_{d}$ is the power response, $T_{p}$ is the load recovery time constant, $\alpha t$ is the transient active loadvoltage dependence, and $\alpha s$ is the steady state active load-voltage dependence:

$$
\begin{aligned}
& T_{p} \dot{x_{p}}=P_{s}(V)-P_{d}, \\
& x_{p}=P_{d}-P_{t}(V), \\
& P_{S}(V)=P_{0}\left(\frac{V}{V_{0}}\right)^{\alpha s}, \\
& P_{t}(V)=P_{0}\left(\frac{V}{V_{0}}\right)^{\alpha t} .
\end{aligned}
$$

\subsubsection{Induction motor (IE)}

The induction motor model refers not only to loads where motors are predominating, but represents loads whose dynamics during starts or stops require reactive power to sustain its operation. To represent the dynamic behavior of the load, the expression shown in Equation (9) is used. The term $T_{L}$ is the mechanical torque on shaft $[p u], w$ represents the angular velocity on shaft $[p u]$, and the coefficients of torque are $a, b$, and $c$ :

$$
T_{L}=a+b w+c w^{2} .
$$

\subsubsection{Frequency-dependent load model (FD)}

The FD model is the general nonlinear exponential voltage-dependent model used in the ZIP load [19] as shown in Equations (10) and (11). Herein, the term $\Delta w$ is the frequency deviation, $k_{P-Q}$ is the active or reactive power percentage, and $\alpha$ and $\beta$ are the exponential coefficients of the active and reactive power, respectively:

$$
\begin{aligned}
& P=\frac{k_{P}}{100}\left(\frac{V}{V_{0}}\right)^{\propto P}(1+\Delta w)^{\beta P}, \\
& Q=\frac{k_{Q}}{100}\left(\frac{V}{V_{0}}\right)^{\propto Q}(1+\Delta w)^{\beta Q} .
\end{aligned}
$$

\subsection{Continuation power flow (CPF) model}

The CPF model is used in this research to find the PV curve because it is more precise with the values obtained in the maximum load points of the system. The methodology uses a predictor-corrector vector to find the solution to the modified power flow equations where the load parameter is included [20] and, thus, the maximum load point is calculated considering (12) and (13). Herein, the term $\theta$ is the vector of voltage angles, $V$ is a vector of voltage magnitudes, $\lambda$ is a vector that represents the parameters that multiply the power of loads, and $\lambda_{\text {Max }}$ is the maximum parameter that multiplies the power of load in the power system.

$$
\begin{aligned}
& F(\theta, V, \lambda)=0, \\
& O \leq \lambda \leq \lambda_{\text {Max }} .
\end{aligned}
$$

Thus, real and reactive powers of loads are modified using the parameter $\lambda$ as shown in (14), where 
$k$ is the constant associated with the rate of power change [20]. The term $P_{i}$ is the new real power of load in bus $i$ and $P_{i}^{0}$ is the initial real power of load connected to bus $i$. These changes are used to find the maximum power that the system can supply to loads and the results can be represented by using PV or PQ curves:

$$
P_{i}=P_{i}^{o}(1+\lambda k)
$$

In this research, voltage stability analysis is performed by evaluating the behavior of the power system after changing some parameters of elements, frequency, or torque. This theory assumes that the parameters vary slowly to identify how and when a system becomes unstable [21].

\section{RESULTS AND ANALYSIS}

\subsection{Study case}

Figure 2 shows the 4-bus test feeder used for the test. This network has a main feeder with a voltage of $18 \mathrm{kV}$ and $50 \mathrm{~Hz}$. This power network considers one load connected to bus 3 , which is modeled as described in the methodology (ZIP, ER, IM, or FD). Two lines are considered in this diagram to separate generation and load and they are modeled as a PI-equivalent circuit. Only one generator will be connected to bus 1 at each simulation and the model depends on the type of machine as previously described in methodology (wind and thermal generators). A power transformer connects the generator in bus 1 and it is modeled as equivalent impedance.

With respect to the wind farm, it consists of 25 turbines of $2 \mathrm{MW}$. The thermal generator has a power of $50 \mathrm{MVA}$, a rating voltage of $18 \mathrm{kV}$, and an armature resistance of $0.01 \mathrm{p} . \mathrm{u}$. as this large power machine involves windings of $100 \mathrm{~m} \Omega$. The leakage reactance was neglected, thus the power losses in the core were not considered as characteristic of thermal generators associated with its high speed. A higher moment of inertia was configured, where the mechanical start time was $12.8 \mathrm{~s}$.

The static ZIP load model was tested with $50 \%$ of the impedance factor, $25 \%$ of the current factor, and $25 \%$ of the power factor. The rated active and reactive powers of the load were considered as $P_{0}=30 \mathrm{MW}$ and $Q_{0}=15 \mathrm{MVar}$, respectively. The induction motor in this document has a nominal power of $20 \mathrm{MVA}$ at $30 \mathrm{kV}$, simulated under a third-order dynamic model. The resistance and reactance of the stator are considered as 0.01 p.u. and 0.06 p.u., respectively. The resistance and reactance in the rotor are considered as 0.02 p.u. and 0.06 p.u., respectively. The magnetization reactance is considered as 3.5 p.u. and the inertia constant is $1.5 \mathrm{kWs} / \mathrm{kVA}$. As the IM is modeled as a speed-dependent load that depends on mechanical torque, the mechanical torque and the moment of inertia are increased with values between 0.5 and 5. In the FD, the real or reactive power percentage was modeled by considering $\alpha$ and $\beta$ with values between 0.5 and 10 . For all cases, the simulation considers changes in power as $0.5,0.99$, and 1.22 with the reference value found in the saddle-node bifurcation.

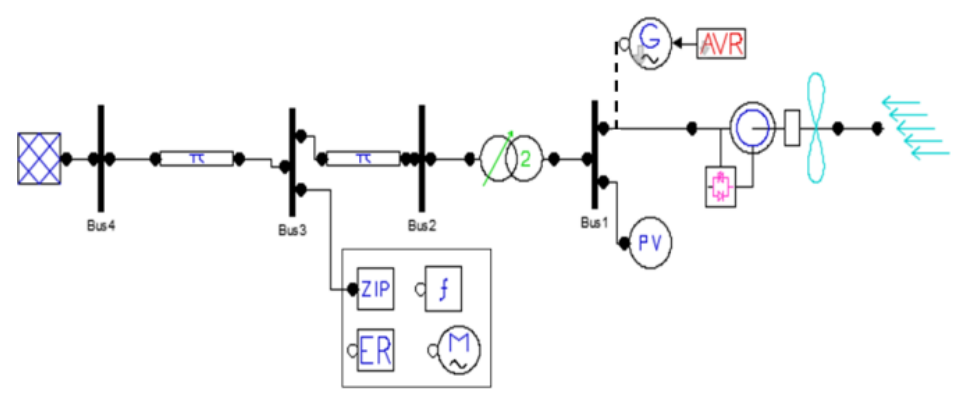

Figure 2. System model of the test that considers the generation and loads models previously described [17]

\subsection{Steady state analysis}

The saddle-node bifurcation was identified by using the continuation power flow method to find the maximum load margin around the nominal power load, considering the different load types. The results are shown in Table 1. 
Table 1. Load Margin Considering Load Type

\begin{tabular}{cc}
\hline Load type & Load margin \\
\hline ZIP & 2.555 \\
ER & 2.706 \\
IM & 2.706 \\
FD & -- \\
\hline
\end{tabular}

After identifying the saddle point as the voltage stability limit, some power variations are performed at $0.5,0.99$, and 1.2 times the maximum power, with the aim of identifying the dynamic behavior of voltage magnitudes in the network. These simulations are carried out by using the CPF to obtain the curves shown in Figure 3. This figure considers the load model connected to bus 3, the power network connected to bus 4 , and PV generation connected to bus 1 .

Figure 3(a) shows the PV curves with a ZIP model. The results show that an increase in the system loadability leads to a reduction of the maximum load parameter $\lambda$ calculated with the CPF. Thus, a smaller power variation leads the power system to a fast voltage collapse because the system is operating close to the voltage limits.

Figure 3(b) shows the PV curves with the ER model or the IM model. The results show the PV curves are similar for both load models, as the continuation power flow does not consider dynamic parameters of load and the increase is performed with static steps. The simulations with the FD model were not succeeded because this model is a frequency-dependent load and the steps of the algorithm do not consider these variations.

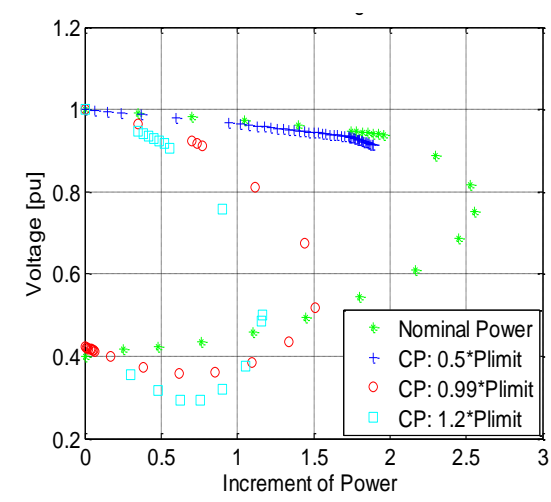

(a)

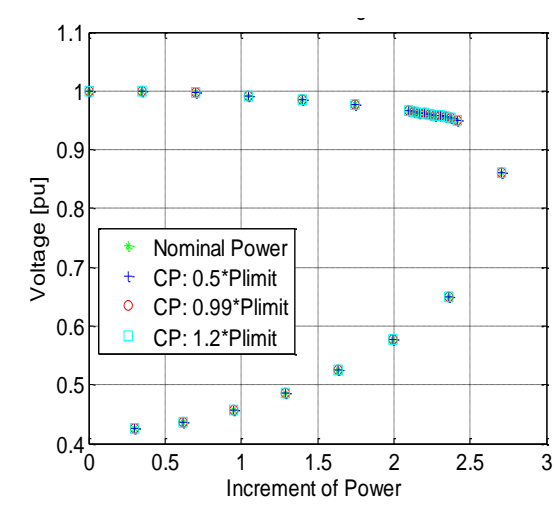

(b)

Figure 3. PV curves for different power loads for the (a) ZIP model and (b) ER and IM models

\subsection{Time-domain simulation}

In [17], some simulations were performed to identify several Hopf bifurcations by using the same test case. In this paper, we focus on the interpretation of the different behaviors of the voltage magnitude with the time-domain simulation, starting from the critical values of the load in the power system. Thus, Figure 4 shows the behavior of the voltage magnitude in buses 1 and 3 when the nominal load is multiplied by 1.8 times and two types of load are connected to bus 3 .

Figure 4(a) shows the response of the system under this operation condition when the load is a ZIP model. The results show that both buses begin with a high voltage magnitude but when the load increases, the DFIG overspeeds and the voltage drops in about 2 seconds. Although the voltage dropped, it did not collapse and the system is capable to stabilize.

Figure 4(b) shows the voltage collapse with the IM load model when the real power is multiplied by the same load factor, as previously defined. This figure shows the bifurcation points when the power changes with a lower value than the maximum power determined previously with the CPF. This point can be associated with the Hopf bifurcation point, because the voltage magnitudes of the buses change abruptly and then reach zero in a few milliseconds. 


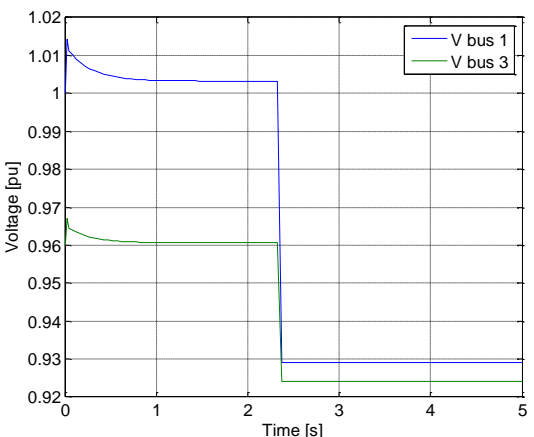

(a)

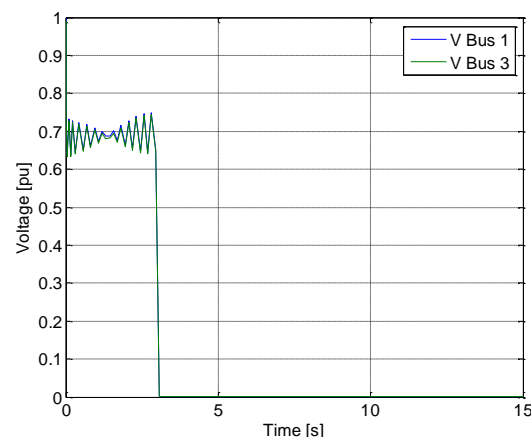

(b)

Figure 4. Voltage magnitudes calculated with time-domain simulations considering (a) ZIP and (b) IM models

Figure 5 shows the voltage magnitudes in bus 3 calculated with time-domain simulations. In these cases, voltage magnitudes are compared when the ZIP or ER load models are connected to the bus 3 and the thermal generator or the wind generators are connected to bus 1. Figure 5(a) shows a voltage collapse when the real power is multiplied by a factor of 1.2, whereas Figure 5(b) shows that the system does not reach voltage collapse. For the thermal generation case, the voltage magnitudes are higher than those obtained with the wind generation case. Figure 5(c) and Figure 5(d) show that the ER load does not present as inconvenient during the operation for the considered thermal and wind generator, i.e., both are capable of maintaining voltage stability despite of the factor multiplied to reach the power limit.

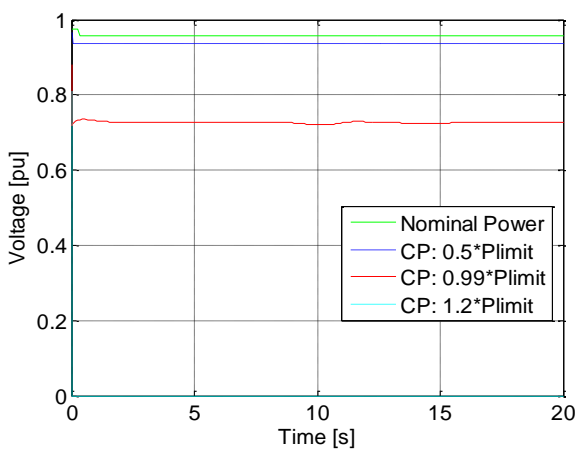

(a)

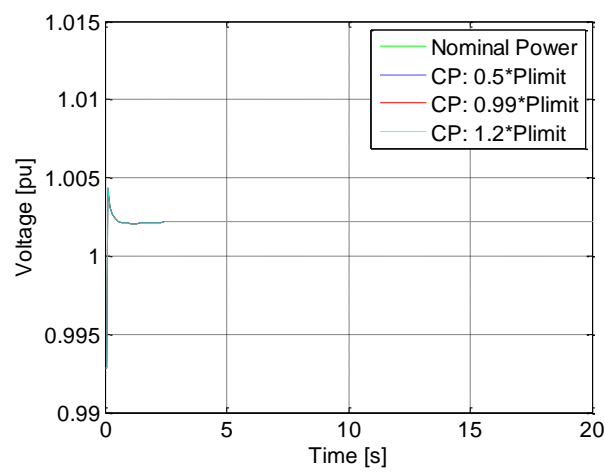

(c)

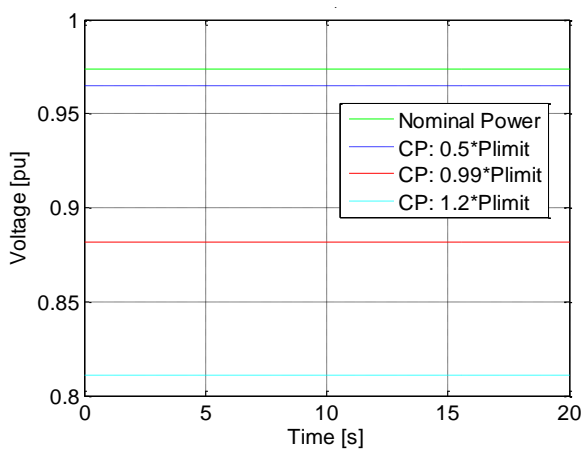

(b)

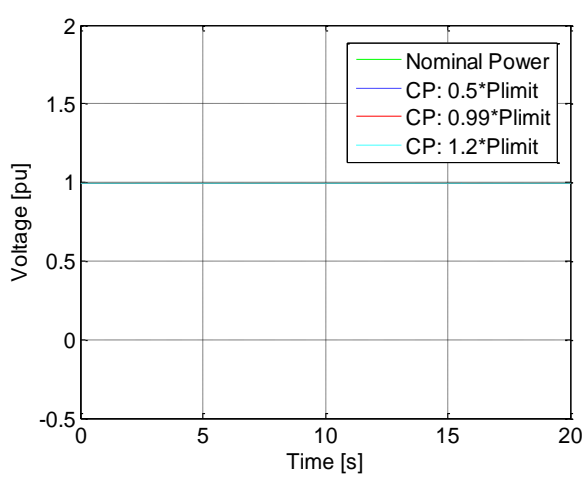

(d)

Figure 5. Time-domain simulation considering different power values with (a) ZIP model and wind generator, (b) ZIP model and thermal generator, (c) ER model and wind generator, and

(d) ER model and thermal generator 
Figure 6 shows the voltage magnitudes in bus 3 calculated with time-domain simulations. In these cases, the IM load is connected to bus 3 and the inertia moment and the torque are changed. The results show that voltage collapses are presented in the system when wind generators are considered in the study. Figure 6(a) and Figure 6(b) show that the voltage magnitudes have different behaviors considering the torque variation. Figure 6(a) shows that the system collapses when the torque increases by a factor of 0.99 and Figure 6(b) shows that the voltages in the three variations have the same behavior and the system stabilizes. Figure 6(c) and Figure 6(d) show large voltage magnitude variations in bus 3, meaning that thermal and wind generators are more sensitive to the increase of inertia. It is noted that the wind generator is unable to respond to a factor that increases seven times, resulting in a voltage collapse; however, the thermal generator oscillates around 10 seconds to the same event, but stabilizes after few seconds.

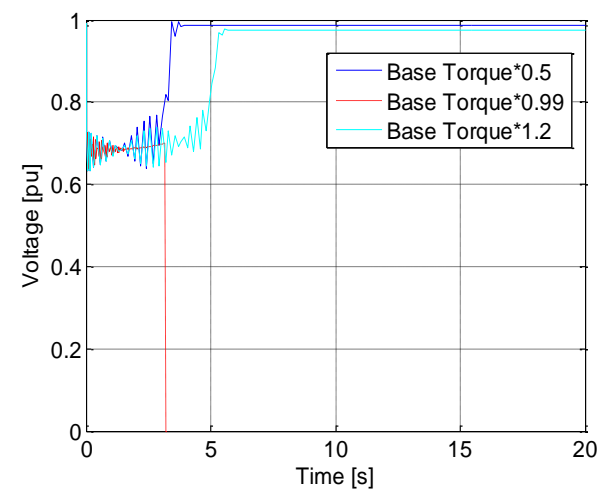

(a)

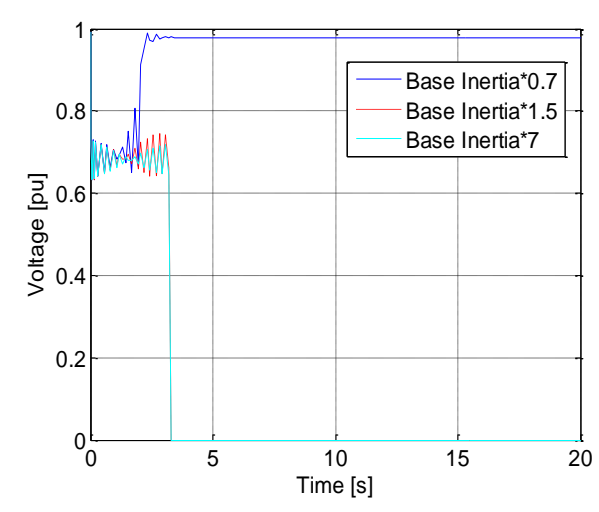

(c)

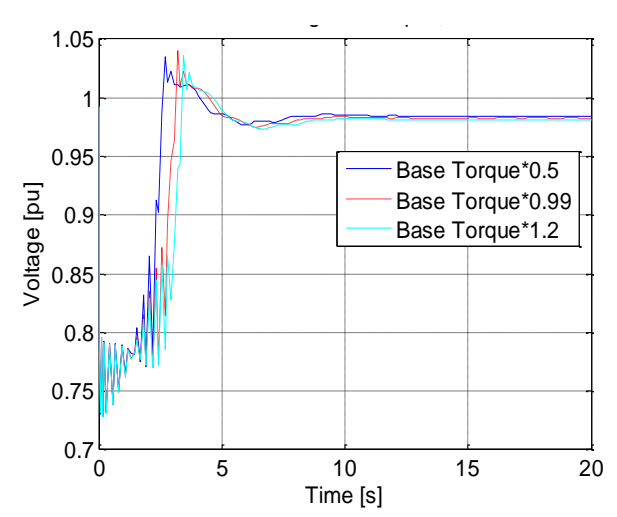

(b)

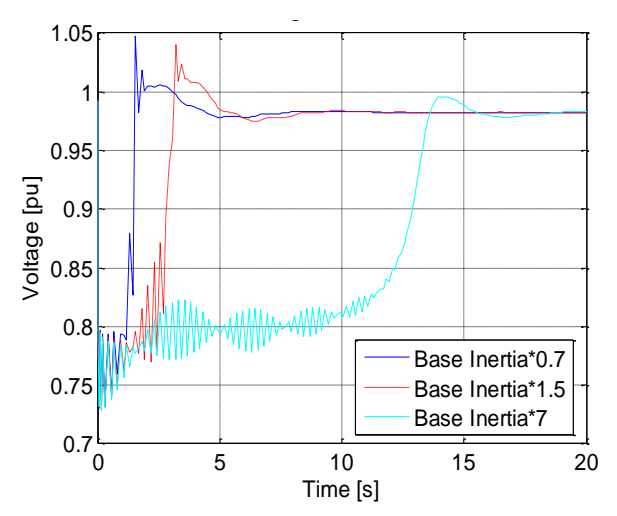

(d)

Figure 6. Time-domain simulation considering IM load model with (a) wind generator and torque variation, (b) thermal generator and torque variation, (c) wind generator and inertia moment variation, and (d) ER model and inertia moment variation

Figure 7 shows the voltage magnitudes in bus 3 calculated with time-domain simulations. In these cases, the FD load is connected to bus 3 and the real or reactive power percentages are changed. The results show that the system is very sensitive to parameter variations and both generators are not able to respond to the load percentage with the FD model and system collapses.

The results show that there is no difference in the PV curves obtained by the CPF for wind and thermal generators when dynamic models are considered, because the CPF does not reflect the dynamic behavior for the static characterization of the PV curve. When nominal power increases closer to the saddlenode bifurcation, the changes around the nominal power are smaller and a reduction of the load factor is presented. Thus, Table 2 summarizes all the results obtained with the time-domain simulations with different parameter variations. 


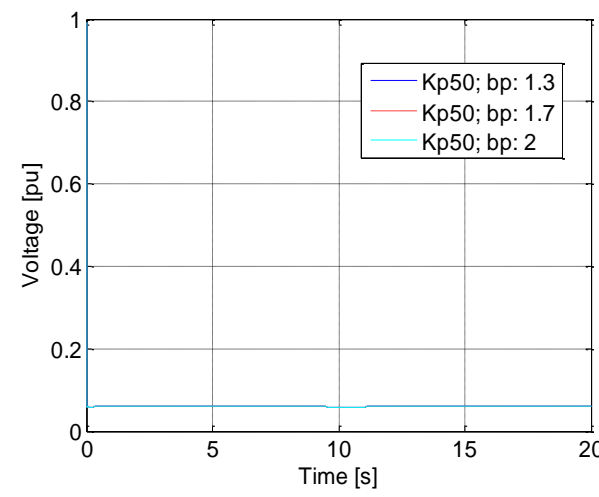

(a)

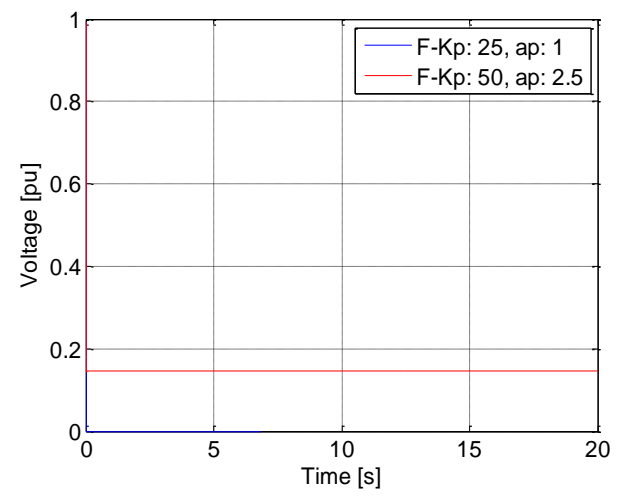

(b)

Figure 7. Time-domain simulation considering an FD model for (a) wind generator and (b) thermal generator

Table 2. Summary of the Results Obtained from the Time-Domain Simulations

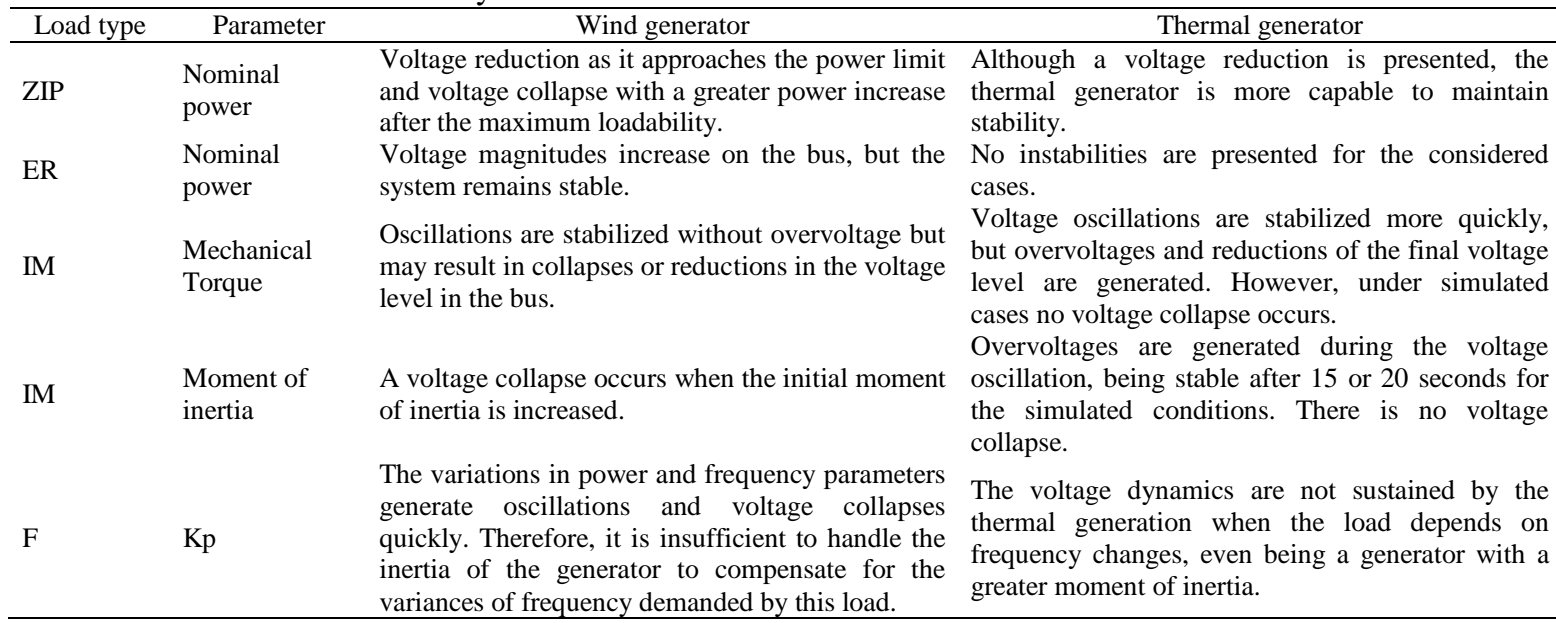

\section{CONCLUSION}

A static and dynamic stability analysis was performed in a 4-bus system with different generation and load types to identify the voltage behavior with parameter variations. PV curves were calculated to find the maximum loadability of the power system (saddle point). Additionally, we performed time-domain simulations considering wind and thermal generators and different load models because PV curves do not consider the type of generation as it is treated as a PV bus. From the results, we found that the exponential recovery load has the widest range of variation in the different cases and improves the voltage magnitudes. With the frequency-dependent load, the margins were unknown because of dynamic load models are not considered by the CPF model. A voltage collapse occurred with the ZIP load model when the power was increased beyond the limit because this static load model allows creating the PV curves. The induction motor generates collapses when increasing the moment of inertia. The ZIP load model with thermal generation does conduct the system to a voltage collapse with the simulated cases and the ER model does not present instabilities with both generators. The motor has a better response to voltage fluctuations and the system is capable to maintain stability with a thermal generator. Finally, the FD load model generates many singularities in the solution at the moment of calculating the response with the time-domain simulation.

\section{ACKNOWLEDGEMENTS}

This project was supported by COLCIENCIAS with the Young Researchers and Innovators Programme 2015 and by the Universidad Nacional de Colombia Sede Medellín with the Project Hermes30667. Authors thank to the Department of Electrical Energy and Automation, and the Applied Technologies Research Group GITA for the support to conduct this project. 


\section{REFERENCES}

[1] P. Kundur, et al., "Definition and Classification of Power System Stability IEEE/CIGRE Joint Task Force on Stability Terms and Definitions," IEEE Trans. Power Syst., vol. 19, no. 3, pp. 1387-1401, 2004.

[2] J. E. Candelo, et al., "Métodos para el Estudio de la Estabilidad de Voltaje en Sistemas de Potencia," Inf. Tecnólogica, vol. 19, pp. 97-110, 2008.

[3] Z. Zeng, et al., "Investigation of Wind Farm on Power System Voltage Stability Based on Bifurcation Theory," in 2009 Asia-Pacific Power and Energy Engineering Conference, pp. 1-4, 2009.

[4] J. Choi and M. Kim, "Multi-Objective Optimization of Voltage-Stability Based on Congestion Management for Integrating Wind Power into the Electricity Market," Appl. Sci., vol. 7, no. 6, pp. 573, 2017.

[5] K. Amarasekara, et al., "Characterisation of long-term voltage stability with variable-speed wind power generation," IET Gener. Transm. Distrib., vol. 11, no. 7, pp. 1848-1855, 2017.

[6] A. Rabiee, et al., "Information gap decision theory for voltage stability constrained OPF considering the uncertainty of multiple wind farms," IET Renew. Power Gener., vol. 11, pp. 585-592, 2016.

[7] N. K. Saxena and A. Kumar, "Analytical comparison of static and dynamic reactive power compensation in isolated wind-diesel system using dynamic load interaction model," Electr. Power Components Syst., vol. 43, no. 5, pp. 508-519, 2015.

[8] R. R. Londero, et al., "Long-Term Voltage Stability Analysis of Variable Speed Wind Generators," IEEE Trans. Power Syst., vol. 30, no. 1, pp. 439-447, 2015.

[9] S. Li, "Sensitivity Model of $L$ Index for Steady-state Voltage Stability of Wind Power Systems with Doubly Fed Induction Generators," Electr. Power Components Syst., vol. 44, no. 18, pp. 2017-2024, 2016.

[10] H. Yue, et al., "Probabilistic evaluation of oscillatory stability margin with large-scale wind generation," in 2013 IEEE PES Asia-Pacific Power and Energy Engineering Conference (APPEEC), pp. 1-6, 2013.

[11] P. Thannimalai, et al., "Voltage Stability Analysis and Stability Improvement of Power System," Int. J. Electr. Comput. Eng., vol. 5, no. 2, pp. 189-197, 2015.

[12] A. M. Thin and N. S. Y. Kyaing, "Performance Analysis of Doubly Fed Induction Generator Using Vector Control Technique,” Int. J. Electr. Comput. Eng., vol. 5, no. 5, pp. 929-938, 2015.

[13] I. M. Wartana, et al., "Optimal Integration of the Renewable Energy to the Grid by Considering Small Signal Stability Constraint,” Int. J. Electr. Comput. Eng., vol. 7, no. 5, pp. 2329-2337, 2017.

[14] A. Perez, et al., "Metodologías utilizadas en la determinación de los parámetros de la máquina síncrona : una aplicación en línea," Tecnura, vol. 11, no. 22, pp. 94-111, 2008.

[15] CIGRE Working Group C4.601, "Modeling and dynamic behavior of wind generation as it relates to power system control and dynamic performance," 2007.

[16] F. Milano, "Assessing adequate voltage stability analysis tools for networks with high wind power penetration," in 2008 Third International Conference on Electric Utility Deregulation and Restructuring and Power Technologies, pp. 2492-2497, 2008.

[17] S. Abdelaziz, et al., "Assessment of wind power penetration level in distribution network with consideration of static, motor and composite loads," in 2014 5th International Renewable Energy Congress (IREC), pp. 1-6, 2014.

[18] D. Karlsson and D. J. Hill, "Modelling and identification of nonlinear dynamic loads in power systems," IEEE Trans. Power Syst., vol. 9, no. 1, pp. 157-166, 1994.

[19] F. Milano, "Power System Modelling and Scripting," Berlin, Heidelberg: Springer Berlin Heidelberg, 2010.

[20] V. Ajjarapu and C. Christy, "The continuation power flow: A tool for steady state voltage stability analysis," IEEE Trans. Power Syst., vol. 7, no. 1, pp. 416-423, 1992.

[21] R. A. Schlueter, et al., "Justification of the voltage stability security assessment and diagnostic procedure using a bifurcation subsystem method," IEEE Trans. Power Syst., vol. 15, no. 3, pp. 1105-1111, 2000.

\section{BIOGRAPHIES OF AUTHORS}

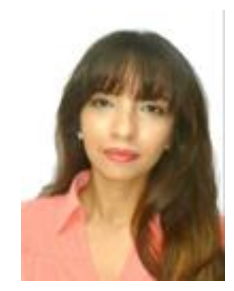

Lina F. Acevedo was born in Barranquilla, Colombia. She received the B.S degree in electrical engineering from Universidad del Norte, Barranquilla, Colombia, in 2014. She is currently pursuing a master's degree in electrical engineering at the Universidad Nacional de Colombia, Medellín. It is linked to the research group on applied technologies (GITA). She develops research in the area of voltage stability, load models, analysis with phasor measurement units, PMU.

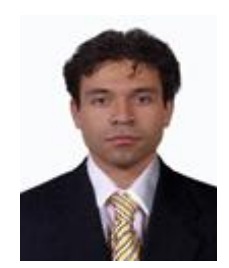

Gilbert L. Bothia was born in Bogota, Colombia. He received the B.S degree in electrical engineering from Universidad Nacional de Colombia, Medellín in 2014. He is currently pursuing a master's degree in electrical engineering at the Universidad Nacional de Colombia, Medellín. It is linked to the research group on applied technologies (GITA). He develops research in the area of voltage stability, load models and fault detection. 


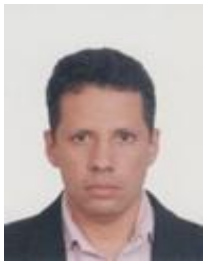

John E. Candelo received his Bs. degree in Electrical Engineering in 2002 and his $\mathrm{PhD}$ in Engineering with emphasis in Electrical Engineering in 2009 from Universidad del Valle, Cali Colombia. His employment experiences include the Empresa de Energ'ia del Pac'ifico EPSA, Universidad del Norte, and Universidad Nacional de Colombia - Sede Medell'nn. He is now an Assistant Professor of the Universidad Nacional de Colombia - Sede Medell'in, Colombia. His research interests include: engineering education; planning, operation and control of power systems; artificial intelligence; and smart grids. ORCID: 0000-0002-9784-9494. 